\title{
L'intérêt de lignées collectées en Haïti pour l'amélioration variétale du haricot grain (Phaseolus vulgaris L)
}

\author{
CM Messiaen \\ INRA-ENSAM, unité de formation et de recherche en biologie végétale, \\ 2, place Viala, 34060 Montpellier cedex 1, France
}

(Reçu le 22 janvier 1992; accepté le 29 mai 1992)

\begin{abstract}
Résumé - Parmi d'autres légumineuses, les grains secs de haricots (Phaseolus vulgaris) tiennent une grande place dans l'alimentation haïtienne. Les types de haricots cultivés en Haïti sont très variés, mais ont une tendance générale à la précocité et à l'indifférence photopériodique. Les paysans cultivent des mélanges de gros grains produits par des plantes naines et de grains moyens et petits produits par des plantes à croissance indéterminée : économie de l'investissement en semences et assurance de meilleure régularité de récolte. Les principaux types variétaux cultivés en Haïti sont décrits, ainsi que les meilleures lignées qui en ont été tirées. Des croisements simples ou complexes faisant intervenir soit exclusivement des lignées haîtiennes, soit aussi des variétés provenant d'autres pays, ont permis d'obtenir des lignées améliorées, intéressant non seulement Haïti, mais cultivables également en Afrique et dans le Midi de la France.
\end{abstract}

\section{haricot / sélection / résistance / adaptation}

Summary - The value of common bean (Phaseolus vulgaris $\mathrm{L}$ ) lines isolated in Haiti for bean varietal improvement. Dry beans and peas belonging to Phaseolus, Vigna, Cajanus and Lablab spp are an important constituent of the diet in Haiti. Of these, the common bean (Phaseolus vulgaris $L$ ) is cultivated on a widespread scale and is the most popular legume. No doubt for historic, sociological and agricultural reasons common beans grown in Haiti are extremely heterogeneous as regards growth habit, seed size and color, but have in common earliness and indifference to photoperiodicity. Haitian farmers grow mixtures of large beans (produced by dwarf plants), and medium-sized or small beans (produced by plants with indeterminate growth) for seed input saving, and to ensure yield regularity. From beans bought in the markets, or from superior plants chosen in the fields several hundred lines were extracted, 50 of which were preserved after severe elimination had been carried out in Guadeloupe (FWI) for powdery mildew susceptibility at the seedling stage or in southern France for summer heat or common mosaic susceptibility. The principal common bean categories grown in Haiti and the best lines found in each category are described:

- large kidney-shaped beans (most of them mottled red) produced by dwarf plants (4 lines are described);

- large ovoid beans (most of them mottled red) produced by dwarf plants ( 1 line described);

- medium-sized black beans, produced by plants with indeterminate growth (4 lines described);

- small-sized beans of various colors, produced by plants with indeterminate growth (4 lines described);

- small-sized uniform red beans produced by plants which become climbing under good fertilization (one line described). Crosses were made in order to obtain still better lines for use in Haiti (and other countries), with tolerance to leaf diseases (such as powdery mildew, rust, and Isariopsis, Chaetoseptoria, Cercospora and Entyloma leaf spots), which in Haiti could result in a yield of 1 tha instead of 0.5 , with 0.30 .30 fertilization, but without fungicide sprays. The general objective was to propose to the farmers a "superior mixture" of the best lines. The programs were as follows: large mottled red-kidney-shaped beans; black beans; small pink or red-mottled beans (the best lines obtained, such as "Salagnac 90" for the first program, have been described). A fourth program aimed at obtaining fresh shell-beans for the FWI: red beans, red or red-striated pods (dwarf or climbing lines following the use in various seasons). Most of these breeding programs involved breeding generations alternated between different climates: Haiti/southern France, Haiti/Guadeloupe, or Haiti/southern France/Guadeloupe. As a consequence of the earliness and day-neutral behaviour of the germplasm used, and also possibly the alternated generations system utilized, the lines resulting from these programs show a wide climatic adaptation. Good results were obtained in some parts of Africa (the highlands of Comoro and the Réunion islands; Burkina-Faso), and southern France where most of them are easily grown and have a satisfactory yield. 


\section{IMPORTANCE DES GRAINS SECS DE LÉGUMINEUSES DANS L'ALIMENTATION DU PEUPLE HAITIEN}

D'après les statistiques officielles, on ensemencerait chaque année en Haïti 100000 ha en haricots grains produisant environ $50000 \mathrm{t}$, soit 10 kg par habitant.

À cette production de grains de Phaseolus vulgaris, on doit ajouter ceux de Phaseolus lunatus, Cajanus cajan, Vigna unguiculata, et de façon plus épisodique Lablab purpureus, Vigna umbellata et Vigna radiata qui amènent la consommation annuelle de grains secs de légumineuses à $15-20 \mathrm{~kg}$, soit $10-14 \mathrm{~g}$ de protéines/ jour, ce qui constitue sans doute environ $20 \%$ ou plus de l'apport protéique.

Parmi toutes ces légumineuses, le haricot commun reste l'espèce dominante et la plus appréciée.

\section{HAITI, CENTRE SECONDAIRE DE DIVERSIFICATION}

\section{Introduction}

D'après les données les plus récentes (Toro et al, 1990) la domestication de Phaseolus vulgaris à partir de la forme sauvage (liane ramifiée, à croissance indéterminée, poussant dans des buissons) aurait pris place en plusieurs lieux différents tout au long de la chaîne des Andes. Les 2 centres de diversification principaux se situent, d'une part au Mexique-Guatemala, où dominent des types indéterminés à entre-nœuds courts, ou volubiles-ramifiés, à grains petits ou moyens, d'autre part au Pérou, où sont apparus des cultivars à gros grains, nains (croissance déterminée) ou volubiles peu ramifiés.

Située au carrefour des routes maritimes aux $X V\|\|^{e}$ et XVIIIe siècles, époque où les corsaires $y$ ramenaient des cargaisons de navires espagnols de toute l'Amérique latine, Haïti a dû connaître de nombreuses occasions d'introduction de grains de Phaseolus vulgaris venant des 2 zones signalées ci-dessus.

Au fur et à mesure de l'accroissement démographique, et du fait de l'acharnement à survivre de ses habitants libérés en 1804 de l'esclavage et de la tutelle coloniale, des pressions de sélection originales se sont exercées au cours des $X \mid X^{e}$ et $X X^{e}$ siècles sur le matériel introduit.
Le résultat se traduit aujourd'hui par une extrême diversité des types variétaux cultivés en Haïti, que n'épuise pas la classification du matériel observé en catégories basées sur le mode de végétation des plantes, la taille, la forme, la couleur et l'ornementation des grains : il s'y ajoute une importante variabilité intracatégories pour la précocité, la vigueur, l'aptitude au rendement avec ou sans fertilisation, et la réaction aux maladies.

\section{Conditions de culture du haricot en Haïti, et leurs conséquences sur les qualités du matériel (fig 1)}

Les dates de semis suivant l'altitude et les saisons sont déterminées par les conditions de température et de pluviométrie favorables à la plante, ainsi que par l'intervention d'un complexe mosaïque dorée/cicadelles (Bean golden mosaic virus, transmis par Bemisia tabaci, et Empoasca krameri), favorisé par les températures élevées. Ce complexe abaisse aux environs de $25^{\circ} \mathrm{C}$ les températures maximales compatibles avec une bonne végétation.

De ce fait, en plaine, les seuls semis possibles se situent en décembre, grâce aux pluies (plaine du cap Haïtien régulièrement soumise aux pluies de "fronts froids"), ou à l'irrigation (plaines de la péninsule du Sud). C'est en plaine que Vigna un-

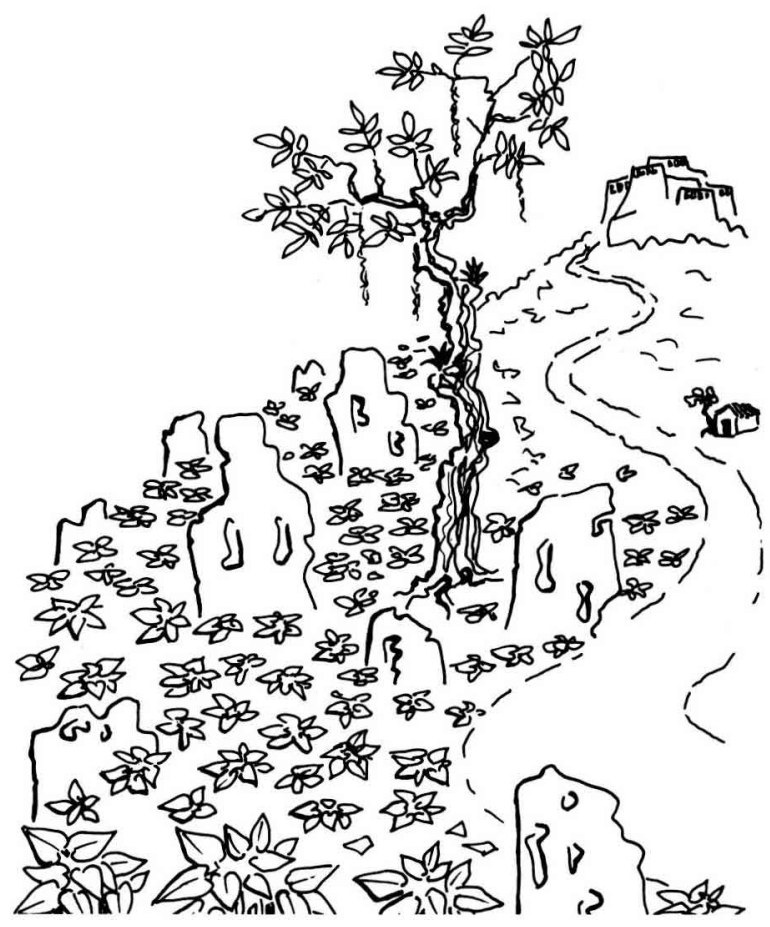

Fig 1. Dessin représentant la situation des cultures de haricots sur une pente abrupte, entre des rocs de calcaire dolomitique, au bord du chemin qui mène à la citadelle du Roi Christophe, en Haïti (novembre 1971). 
guiculata et Cajanus cajan supplantent le haricot pour la production de grains secs.

Aux altitudes moyennes (400-500 m) les semis sont possibles en février-mars et en octobre, les semis de juillet restant interdits.

À $800-900 \mathrm{~m}$, on peut semer 3 fois dans l'année; fin février, juillet et octobre, chaque récolte fournissant les semences à la culture suivante.

Au cours des siècles, il est probable que les paysans haïtiens, aussi bien dans les mornes pour réaliser 2-3 semis par an, qu'en plaine pour arriver à la floraison avant que ne se manifestent mosaïque dorée et cicadelles, ont exercé une pression de sélection pour la précocité. Par ailleurs, la situation déjà nordique du pays (18$20^{\circ} \mathrm{N}$ ) entraîne une variation notable de la longueur du jour au cours des saisons (10 h 30-13 h 30).

Le matériel génétique haïtien se caractérise donc assez logiquement, malgré sa diversité, par une tendance générale à la précocité et à l'indifférence photopériodique.

Les rendements en culture traditionnelle restent assez faibles : de l'ordre de 0,5 t/ha en cultures pures semées à très forte densité (400600000 grains/ha), et de 0,2 à $0,3 \mathrm{t} / \mathrm{ha}$ en culture associée avec, par exemple, maïs et patates douces dans les mornes de la péninsule du Sud.

Les facteurs limitants sont de natures diverses: - édaphique : sols pauvres, parfois trop acides, carencés en acide phosphorique et en potasse, insuffisamment pourvus en matière organique. Ces facteurs limitent la nodulation et la fixation de l'azote, bien que le Rhizobium phaseoli soit généralement présent dans les sols haïtiens;

- parasitaire : les maladies cryptogamiques foliaires sont le facteur limitant majeur; on peut citer : - l'ö̈dium, Erysiphe polygoni (Messiaen et al, 1989; Pauvert, 1989);

- la rouille, Uromyces phaseoli;

- l'anthracnose, Colletotrichum lindemuthianum, ainsi que Phaeoisariopsis griseola, Chaetoseptoria wellmanii, Cercospora castellani, Entyloma petuniae (Belliard-Alonzo, 1971; Schwartz et Galvez, 1978).

Sans oublier les cicadelles mentionnées cidessus et les bruches sur grains en conservation, l'insecte le plus nuisible en conditions normales de culture est Hedylepta indicata, dont la chenille replie une feuille ou en attache 2 ensemble par des fils de soie avant de les dévorer.

Des essais, réalisés par l'équipe agronomique du centre de recherche et développement de Madian-Salagnac (péninsule du Sud) dans les an- nées 1970, ont mis en évidence l'interaction des facteurs édaphiques et parasitaires sur les rendements. Une protection fongicide (2 traitements avec un mélange manèbe + thiophanate) plus une fumure phosphopotassique 0-30-30 (cendre de bois plus superphosphate) font passer les rendements de 0,5 à 1 ou 1,2 t/ha, alors que l'un ou l'autre traitement appliqués isolément restent sans effet.

Un complément azoté (fumier, ou usage d'un engrais complet) faisant passer la fertilisation à 30-60-60 permet d'atteindre 1,5 tha. Par contre, une dose de 60 unités d'azote n'apporte pas de bénéfice supplémentaire, et entraîne l'apparition de Xanthomonas phaseoli. Ces résultats ont été obtenus avec des lots de semences de type traditionnel, non homogènes.

Les paysans haïtiens cultivent en effet des mélanges variétaux comprenant 2 à 3 catégories dominantes, accompagnées de 5 à 10\% de minoritaires très variés (fig 2). Ces mélanges sont binaires dans la région du cap Haïtien : pois Descayettes + pois Pistache; ternaires dans la péninsule du Sud: pois Rouge + pois Noir + Ti Pémet.

Cette tradition de semis de lots hétérogènes et, en particulier le mélange de types nains à gros grain (faible taux de multiplication, floraison groupée) et de types à croissance indéterminée, grains moyens ou petits (à fort taux de multiplication, floraison échelonnée) s'explique chez le paysan par le souci contradictoire de :

- récolter pour la vente au marché des grains qui atteignent un prix élevé (gros grains rouges panachés, produits par les plantes naines);

- d'économiser sur l'investissement en semences, en améliorant les chances de réussite, les grains moyens ou petits, produits par des plantes à croissance indéterminée, se partageant entre vente et autoconsommation.

En effet, la meilleure tolérance des pois Noirs aux périodes chaudes et pluvieuses, la meilleure régularité de récolte des plantes à floraison échelonnée sont bien perçues par les cultivateurs.

\section{RECHERCHE DE LIGNÉES SUPÉRIEURES DANS LE MATÉRIEL HAITIEN}

\section{Isolement des lignées}

À partir de ces lots hétérogènes, nous avons essayé d'isoler des lignées supérieures pour le rendement et la tolérance aux maladies foliaires. En effet, jusqu'à une époque récente, les pay- 
sans haïtiens ne pouvaient guère avoir accès au matériel de pulvérisation, même manuel, alors que la réalisation de fumures modestes de type 0-30-30 semblait plus facilement accessible. Avec des lignées tolérantes aux maladies foliaires, le doublement des récoltes grâce à ce type de fumure pourrait être obtenu sans traitement fongicide (le cas de l'anthracnose est particulier, car elle peut être tenue en respect par un traitement des semences avec un fongicide de type "benzimidazole», que pratique le centre de Madian-Salagnac).

Suivant les régions, en Haïti, les hybridations accidentelles sont absentes (ex: Salagnac), ou ne dépassent pas 1\% (Camperrin). On obtient donc, dans la plupart des cas, des lignées homogènes en conservant la descendance d'un seul grain (lots achetés au marché), ou mieux encore, d'une seule plante repérée chez le paysan pour son état sanitaire, sa vigueur et le nombre de ses gousses : cette dernière méthode a été pratiquée en 1975 par $V$ de Reynal (Centre Madian-Salagnac) sur les conseils de $H$ Bannerot (INRA, Versailles).

Plusieurs centaines de lignées ont été ainsi introduites de 1971 à 1985, soit en Guadeloupe, où le tri initial était effectué sur la résistance à l'oïdium au stade plantule par $G$ Jacqua (INRA, Antilles-Guyane) soit à Montpellier (INRA-ENSA) où une très importante élimination était assurée par la mosaïque commune (BCMV), virus peu agressif en Haïti : la majorité des plantes issues de lots introduits à Montpellier étaient soit stérilisées par une mosaïque cloquée intense, soit nécrosées par le symptôme "black-root" (hypersensibilité généralisée).

Des générations alternées Montpellier-Haïti, Guadeloupe-Haïti, et souvent GuadeloupeMontpellier-Haïti faisaient suite à ce tri initial. Une cinquantaine de lignées ont été ainsi conservées dont 45 ont été remises au CIAT (Centro international de agronomia tropical, Cali, Colombie) en 1982. Une trentaine sont conservées à I'INRA de Versailles à $-18^{\circ} \mathrm{C}$.

\section{Principaux types variétaux cultivés en Haïti, et lignées les plus intéressantes tirées de chacun d'entre eux}

La liste ci-dessous ne prétend pas être exhaustive, du fait de la présence dans les mélanges des types minoritaires évoqués ci-dessus. et que tous les marchés, toutes les régions du pays n'ont pas été explorés: nous n'avons fait qu'effleurer la masse considérable du germoplasme haïtien.

\section{Grains allongés de fort calibre (300-400 mg), plantes naines, en général très sensibles à l'oïdium}

La coloration dominante dans cette catégorie est panaché rouge sur fond clair, qualité la plus demandée dans la péninsule du sud et appelée Manzé Joute* sur le marché de Jacmel. On trouve aussi des panachés violets sur fond clair (marché de Camperrin) et. comme minoritaires, des grains roses striés de rouge, beige striés de noir, marron clair ou noir brillant.

Les caractères Panaché et Strié correspondent aux linkats C-Rm et C-Rst décrits par Prakken (1972). Dans les 2 cas, les lignées pures produisent $1-5 \%$ de grains plus foncés, très apparents dans le cas de C-Rst. Ceux-ci donnent une descendance conforme au type si on les sème séparément.

Les lignées les plus intéressantes tirées de cette catégorie ont été :

- Kenscoff I (marché de Kenscoff, 1971), à grains roses striés de rouge, $320 \mathrm{mg}$, gousses étroits et longues, tolérante à l'oïdium, très précoce (60 j en Guadeloupe);

- Salagnac 86 (prospection de Reynal-Jacqua, 1975) : à grains panachés rouge-blanc, $380 \mathrm{mg}$, hautement tolérante à l'oïdium, tolérante à la rouille et aux maladies foliaires mineures, moyennement précoce (68 j en Guadeloupe);

- Azaël 175A (lignée isolée par l'agronome haïtien A Azaël) : à grains rouges tachetés de blanc (allèle de la série $\mathrm{C}-\mathrm{R}$, mais différent de $\mathrm{C}-\mathrm{Rm}$ et C-Rst), $380 \mathrm{mg}$, pas de résistance particulière aux maladies, moyennement précoce (70 j en Guadeloupe);

- Camperrin 2.1 (marché de Camperrin, 1978): grains panachés violets sur fond clair, $380 \mathrm{mg}$, port nain buissonnant. pas de résistance particulière aux maladies. movennement précoce $(70 \mathrm{j}$ en Guadeloupe).

\section{Grains ronds de fort calibre, plantes naines}

C'est surtout dans le Nord que l'on trouve ce type, comme l'une des 2 catégories dominantes dans les mélanges. sous le nom de pois Descayettes. On le retrouve comme minoritaire dans les autres régions. Nous avons retenu dans cette catégorie : 
- Salagnac 92 (prospection de Reynal-Jacqua, 1976), grains ronds panachés rouge sur fond clair, $410 \mathrm{mg}$, plantes vigoureuses, ramifiées et assez hautes, légèrement tolérantes à l'oïdium. Précocité moyenne (68 j en Guadeloupe).

\section{Grains noirs de calibre moyen ou petit (180- $250 \mathrm{mg}$ ) plantes à croissance indéterminée}

Les lignées de ce type ont en général des grains mats, ce qui entraîne une perte rapide de l'anthocyane dans l'eau de cuisson, colorant le riz en bleu. On les fait donc en général cuire à part, en "sauce pois noirs" dont on arrose le riz ou le sorgho, contrairement aux haricots des autres catégories, en général cuits en mélange avec le riz. Elles sont très généralement peu sensibles à l'oïdium au stade adulte, bien que sensibles au stade plantule - et, au contraire, assez sensibles à la rouille. Leur précocité est variable : $65-78 \mathrm{j}$ en Guadeloupe.

Nous avons retenu dans cette catégorie :

- Kenscoff B (marché de Kenscoff, 1971) 250 $\mathrm{mg}$;

- Nicanor 2.4 (d'un lot cultivé par un paysan de Salagnac) $190 \mathrm{mg}$;

- B.789 (isolée par H Bannerot d'un lot acheté à Petite Rivière de Nippes). Résistance à l'anthracnose de type Cornell (mais des souches de Colletotrichum lindemuthianum surmontant cette résistance ont été isolées par Bannerot à partir d'échantillons provenant d'Haïti), la plus précoce (65 j en Guadeloupe), 225 mg;

- Haïti $D$ (isolée par $F$ Kaan d'un lot du marché de Port-au-Prince 1977), la plus tardive (78 j en Guadeloupe, analogue à des haricots noirs cultivés à Cuba), $185 \mathrm{mg}$.

Aucune de ces lignées n'a semblé totalement satisfaisante aux agronomes du centre MadianSalagnac, qui leur ont préféré une population Mersant, prélevée par P Mathieu chez des paysans très isolés sur les flancs du Mont Macaya. Cette population, hétérogène en particulier pour la coloration des gousses (incolores, striées de violet, ou violettes) présentait, dans son ensemble, un niveau de résistance à la rouille supérieur à celui des lignées énumérées ci-dessus. Elle a été reprise en lignées parallèlement en Guadeloupe, et en Haïti par A Laraque. Sur la quarantaine de lignées isolées, 10 ont été choi- sies pour être distribuées en mélange par le centre Madian-Salagnac.

\section{Petits grains de coloration variée (150- $250 \mathrm{mg}$ ) plantes à croissance indéterminée}

Cette catégorie est sans doute la plus hétérogène. Le type Ti Pemet à petits grains arrondis mauves unis, plantules à hypocotyle violet, peut représenter jusqu'à $30 \%$ des mélanges ternaires cultivés dans la péninsule du Sud. Consommé par les populations qui le cultivent, il arrive très peu sur les marchés. Dix pour cent des mêmes mélanges peuvent être constitués de petits grains rouges panachés mais dont la panachure répond à un autre mécanisme que celui du linkat $\mathrm{C}-\mathrm{Rm}$ : les lignées pures produisent une certaine proportion de grains clairs pointillés de rouge, et leur hybridation avec des lignées $\mathrm{C}$ Rst conduit à des disjonctions d'une effarante complexité.

Outre ces 2 types les plus fréquents, on trouve aussi dans cette catégorie des grains panachés de violet, des grains striés de rose sur fond blanc, striés de noir sur fond beige, ainsi que des petits grains blancs ressemblant à ceux de la variété américaine Michelite, recherchés dans des conditions particulières : repas du Mercredi des Cendres, ou pour le régime des personnes souffrant d'hypertension*.

Nous avons retenu dans cette catégorie les lignées:

- B.801 (extraite par Bannerot d'un lot acheté à Petite Rivière de Nippes, 1975), grains de type "Ti Pemet", $180 \mathrm{mg}$, précocité moyenne, plantes vigoureuses et productives, peu sensibles à l'oïdium en fin de végétation;

- Salagnac 126 (prospection de Reynal-Jacqua, 1976), également de type Ti Pemet, $180 \mathrm{mg}$, mais plantes moins vigoureuses que celles de B.801, très sensibles à la mosaïque commune, tolérantes à l'oïdium dès le stade plantule;

- Salagnac 120 (de Reynal-Jacqua, 1976), à grains rouges panachés, $120 \mathrm{mg}$, plantes de petite taille, résistantes à l'oüdium dès le stade plantule, sensibles au black-root;

- Salagnac 131 (de Reynal-Jacqua, 1976), à grains blancs striés de rose, $250 \mathrm{mg}$, sensible à la mosaïque commune, immunité à l'oïdium à tous les stades.

\footnotetext{
"C'est en Haïti un "métier», souvent exercé par des femmes-chefs de famille, aidées par les enfants, que de trier en couleurs homogènes des lots reçus en mélange. La rumeur récente accusant les haricots rouges d'aggraver l'hypertension est sans doute sans fondement.
} 
Grains rouges unis de taille moyenne (200$300 \mathrm{mg}$ ), plantes à croissance indéterminée pouvant devenir volubiles

Pratiquement absente dans le Sud du pays cette catégorie participe, sous le nom de pois Pistache aux mélanges binaires cultivés dans la plaine du Nord. Cette appellation est sans doute due au fait que les grains, très près les uns des autres dans les gousses courtes, présentent une forme tronquée aux 2 extrémités, comme des graines d'Arachide dans leur coque. On peut aussi rattacher à cette catégorie le pois 7 semaines du plateau Seguin, que nous n'avons pas pu conserver à cause de sa très grande sensibilité à la mosaïque commune (mosaïque nécrosante).

Nous avons retenu dans cette catégorie la lignée:

- Cap Haïtien 9.4 (prospection dans la plaine du Nord 1978), à grains rouges de $280 \mathrm{mg}$, se comportant en Guadeloupe comme volubile en toutes saisons sous bonne fertilisation. La récolte s'échelonne entre le $60^{\mathrm{e}}$ et le $75^{\mathrm{e}} \mathrm{j}$; cette lignée se montre aussi productive et de $15 \mathrm{j}$ plus précoce que la meilleure lignée volubile à grain rouge reçue du CIAT.

\section{CONCLUSION}

Parmi les lignées énumérées ci-dessus, seules Salagnac 86 et les 10 lignées distribuées en mélange issues de Mersant se sont prêtées à une diffusion directe auprès des paysans de la péninsule du Sud, auprès desquels s'exerce l'action de développement du centre Madian-Salagnac.

Cependant, Salagnac 86 malgré toutes ses qualités qui permettaient la réalisation de l'objectif « 1,0 à 1,2 tha avec une fertilisation $0-30-30$, sans traitement fongicide" (à condition d'utiliser des semences indemnes d'anthracnose, ou traitées au bénomyl), se révélait insuffisamment coloré pour être vendu au meilleur prix sur les marchés.

Cette constatation, et l'absence d'un type à petit grain rouge ou rose totalement satisfaisant, nous ont entraînés dans des programmes de croisement destinés:

- soit à fournir aux paysans haïtiens de la péninsule du Sud un mélange supérieur,

- soit à tirer parti des qualités de précocité et de rusticité des lignées isolées en Haïti pour obtenir des variétés à large adaptation pouvant être cultivés dans divers pays tropicaux ou tempérés.

\section{PROGRAMMES DE CROISEMENTS ET DE SÉLECTION: RÉSULTATS OBTENUS}

\section{Méthodes}

Ces programmes sont restés très simples, et, sauf dans un cas particulier, n'ont pas le plus souvent dépassé le croisement entre 2 parents suivi de tri dans la descendance, ou des rétrocroisements ne se prolongeant pas au-delà de $2-3$ cycles.

Nous avons soumis, quand cela était matériellement possible, les descendances issues de ces croisements à une politique de générations alternées sous climats différents: de 1976 à 1981 entre Montpellier (France) et Haïti de 1981 à 1987 entre Haîti et Guadeloupe.

Avec le concours de $V$ Reynal et $P$ Lucas, nous avons systématisé cette pratique pendant la période 1976-1981 en pratiquant, chaque année et sans aucun traitement pesticide:

- un semis au 1er mai, à Montpellier, sous fertilisation faible (cendre de bois), avec comme facteurs sélectifs : la mosaïque commune, et des températures diurnes dépassant $30^{\circ} \mathrm{C}$ à partir de la floraison;

- un semis au 1 er octobre, en Haïti, sous forte fertilisation (40-80-80), pour faire s'exprimer les maladies foliaires.

\section{Programme «Gros grains rouges panachés à destination d'Haïti, plantes naines»}

Notre but était d'améliorer et d'intensifier la coloration des meilleures lignées dont nous disposions: Kenscoff I à partir de 1974, et Salagnac 86 à partir de 1979. Nous avons utilisé, en plus de nos lignées haïtiennes : Constanza 1, variété d'origine guatémaltèque cultivée en République Dominicaine, tardive, à gros grains rouges panachés, résistante à la rouille mais très sensible à l'oïdium.

Nous pouvons citer, par ordre d'obtention les lignées :

- $(C 1 \times$ l) 4.3, lignée issue de : (Constanza $1 \times$ Kenscoff I), à gros grains rouges panachés sur fond clair, $400 \mathrm{mg}$, précocité moyenne (65 j en Guadeloupe), tolérance moyenne à l'oïdium;

- Kenscoff Super issue des rétrocroisements: [(Constanza $1 \times$ Kenscoff I) $\times$ Kenscoff I] $\times$ Kenscoff I, à grains rouges panachés sur fond rose, $330 \mathrm{mg}$, très précoce (60 j en Guadeloupe), bonne tolérance à l'oïdium; 
- Salagnac 90, issue des rétrocroisements : [(C1 x I)4.3 x Salagnac 86] x Salagnac 86 , à grains rouges panachés sur fond rose, $380 \mathrm{mg}$, haute tolérance à l'oïdium, tolérance à la rouille et aux taches foliaires mineures; son comportement est analogue à celui de Salagnac 86 , en particulier pour la précocité (65 j en Guadeloupe). Cette lignée a perdu le gène dominant de résistance à la race $O$ d'oïdium de Salagnac 86 , ce qui ne semble pas amener d'inconvénient dans la pratique.

- Azaël $\times S 86^{2}$, issue des rétrocroissements: (Azaël 175A x Salagnac 86) x Salagnac 86, elle aussi très voisine de Salagnac 86 , mais ayant gardé la coloration originale du grain d'Azaël 175A.

La coloration des grains de Kenscoff super et de Salagnac 90 était inédite sur les marchés haïtiens (elle reconstitue celle des Marlat réunionnais) (fig 3). Les consommateurs l'ont beaucoup appréciée, et Salagnac 90 supplante aujourd'hui en Haîti Salagnac 86 (la coloration rouge uni est au contraire peu estimée en Haîti, elle rappelle de mauvais souvenirs : dons des Etats-Unis à la suite de cyclones ou de famines, constitués de haricots Red Kidney qui, essayés comme semences, se révèlèrent très sensibles à la rouille et à l'oïdium).

\section{Programme "Grains noirs, plantes à croissance indéterminée, à destination d'Haïti»}

Avant que les agronomes du centre MadianSalagnac n'aient mis la main sur la population Mersant, nous avions obtenu à partir du croisement : (B.809 x Salagnac 131) la lignée Salagnac 59 à grains noirs, assez gros $(280 \mathrm{mg})$, très précoce (60 j en Guadeloupe), peu encombrante en culture intensive malgré son port indéterminé. Sa résistance à l'oïdium est presque égale à celle de Salagnac 131, mais sa tolérance à la rouille est inférieure à celle des lignées Mersant. Son usage en culture intensive peut cependant être envisagé moyennant un traitement fongicide peu avant la floraison (mancozèbe ou fongicide du groupe «inhibiteurs de synthèse de l'ergostérol»).

\section{Programme "Petits grains rouges ou roses, plantes à croissance indéterminée, à destination d'Haïti»}

Pour ce programme nous avons débuté par des croisements simples, comme (Salagnac $131 \times$
Salagnac 120), puis sous l'influence de $G$ Fouilloux (1978) nous nous sommes engagés dans des croisements complexes de 4 ou 5 géniteurs, dont le plus compliqué fut, avec $S$ pour Salagnac :

$[(\mathrm{S} 92 \times \mathrm{S} 126) \times(\mathrm{S} 131 \times \mathrm{S} 120)] \times[(\mathrm{S} 131 \times \mathrm{S} 120)$ $x$ B801]

Les descendances de ces croisements ont été suivies en Guadeloupe en sélection généalogique, aboutissant à des lignées dénommées $131 \times 120$ (grains rouges panachés), Cx (issues des croisements complexes, à grains roses striés de rouge) ou Dx (issues des croisements complexes, à grains rouges panachés).

De son côté, en Haïti, A Laraque appliquait à la descendance des croisements complexes, confondue en un seul lot, un système de «bulk sélectif" : multiplication en mélange pendant 6 générations ( 2 ans), avec à chaque fois élimination des plantes chétives ou sensibles aux maladies foliaires, suivie d'une extraction de lignées Bx parmi lesquelles il a préféré retenir, à la demande des paysans, celles qui produisaient des grains rouges panachés.

En 1988, le centre Madian-Salagnac distribuait comme $3^{e}$ composante d'un «mélange supérieur', un mélange des lignées (131 $x$ 120).12, $B \times 27$ et $D \times 19$. Cette politique de distribution de lignées en mélange correspond, comme pour celles issues de Mersant, au souci de réduire la vulnérabilité génétique.

\section{Programme "Grains rouge à écosser", à destination des Antilles françaises}

La consommation de haricots grains rouges aux Antilles françaises, assurée par l'importation de Red Kidney américains, atteint aussi environ 10 kg par habitant et par an.

Le climat de ces îles ne présente qu'une brève période (durant le Carême) où la sécheresse soit suffisamment sûre pour programmer une récolte de haricots secs : du 10 février au 20 mars (aux autres périodes de l'année, nos générations de sélection en Guadeloupe étaient récoltées en bottillons et suspendues sous hangar pour le séchage). Par contre, tout au long de l'année, il est possible de programmer une récolte de grains frais à écosser. II est préférable d'utiliser des variétés volubiles aux périodes les plus pluvieuses de l'année (mai, aoûtnovembre) pour éviter les attaques de rhizoctone foliaire (Rhizoctonia solani AG1 microsclerotia). Cette spéculation conduisait à ajouter au 
souci de coloration du grain (de préférence rouge uni) celui de l'esthétique de la gousse, de préférence striée de rouge ou rouge uni. Nous avons utilisé comme géniteurs dans ce programme, outre Kenscoff super et Salagnac 86, les lignées: Caricosse (volubile) et Caricosse nain obtenues précédemment par $G$ Anais et $C$ Vincent à partir du croisement (Scabiola rossa $x$ Red Kidney) et la lignée Épicerie de Sault (Le Red Kidney utilisé par Vincent avait été retrouvé cultivé en Guadeloupe à Vieux Habitants, sans doute à partir de lots d'importation. "Scabiola rossa" est une variété volubile italienne à gousses striées de rouge, grains gris striés de violet. Épicerie de Sault, lignée volubile, à gousses rouges unies, grains beiges striés de marron, est issue de gousses à écosser achetées dans une épicerie de Haute-Provence).

Du croisement : (Épicerie de Sault $\times$ Salagnac 86) $x$ Kenscoff super, ont été tirées des lignées volubiles GR et des lignées naines NR.

On a ensuite réalisé les croisements :

Caricosse $\times \mathrm{GR} \longrightarrow$ Lignées CGR (volubiles)

Caricosse nain $\times \mathrm{NR} \longrightarrow$ Lignées CNR (naines)

Parmi les lignées obtenues, nous pouvons citer :

- CNR44 : grains rouges unis, gousses incolores (c'est-à-dire vertes unies puis jaunes à maturité) hôte différentiel des races 0 et 1 d'oïdium (cette lignée a conservé le gène de résistance à la race " $O$ " de Kenscoff super et Salagnac 86, mais perdu leur résistance polygénique aux 2 races).

- CNR11 : grains rouges striés de noir, gousses rouges unies, tolérance moyenne à l'oïdium (fig 4);

- CNR28 : grains rouges striés de rouge sombre, gousses rouges unies, tolérance moyenne à l'oïdium;

- CGRD : grains rouges unis, gousses incolores, tolérance moyenne à l'oïdium;

- CGRI : grains rouges striés de noir, gousses striées de rouge, tolérance moyenne à l'oïdium.

Parmi toutes les lignées issues de ces croisements (ainsi que dans d'autres croisements) il apparaît que les caractères "gousse rouge unie" ou "gousse striée de rouge" sont très étroitement liés au linkat CRst : il est impossible de combiner "grain uni» (C-R) ou «grain panaché" (C-Rm) avec la coloration rouge ou la striation de la gousse : les grains sont obligatoirement striés, quelles qu'en soient les couleurs (ex: "Scabiola rossa", grains gris striés de violet, "Épicerie de Sault", grains beiges striés de marron, "Coco nain rose d'Eyragues", grains blancs striés de rouge, ou nos obtentions à grain rouge strié de noir ou de rouge sombre).

\section{APTITUDE AU RENDEMENT DES MEILLEURES LIGNÉES HAITIENNES ET DE LEURS DESCENDANTS EVALUÉE EN GUADELOUPE}

Dans les essais réalisés par $C$ Vincent et son équipe à I'INRA-Antilles-Guyane, particulièrement soignés pour leur fertilisation : apport total de l'ordre de 40-80-60, avec apport de scories Thomas ou de phosphate tricalcique dans le sillon de plantation, puis d'engrais complet le long des lignes en cours de végétation, nous avons obtenu dans les essais réalisés de 1984 à 1987 des rendements de l'ordre de :

1,4-1,6 tha pour Kenscoff I et Kenscoff super

$1,5-1,7$ tha pour $\left(C_{1} x l\right) 4.3$

$1,7-1,8$ tha pour salagnac 90

1,6-1,7 tha pour CNR.11, CNR.44, CNR.28

(variétés naines)

1,7-1,9 t/ha pour Nicanor 2.4, Salagnac 59 et le mélange des lignées Mersant

$1,3-1,4$ tha pour Salagnac 120 et Salagnac 131

$1,6-1,8$ tha pour B 801

$1,9-2,1$ tha pour les meilleurs $(131 \times 120)$ et $C x$

(variétés à port indéterminé)

1,7-2 t/ha pour CGR.l et CGR.D

2,3-2,6 t/ha pour Cap Haïtien 9.4

(variétés volubiles)

$\mathrm{Au}$ vu de ces résultats nous avons entrepris un programme visant à réunir les caractères "gros grains" et "port indéterminé", à partir d'un croisement entre un des "CNR" et la variété brésilienne "Avermelhado" (variété à port indéterminé produisant les plus gros grains que nous ayons pu trouver). Ce programme est aujourd'hui en sommeil.

À titre de comparaison, cultivées dans les mêmes conditions, les meilleures variétés de Vigna unguiculata issues du programme de sélection réalisé par $H$ Touvin et $J$ Félicité (INRA Antilles-Guyane) nous donnaient 2,1 à 2,3 tha avec des grains de 110 à $150 \mathrm{mg}$. Cela conduit, en Guadeloupe, sous des températures comprises entre 18 et $31^{\circ} \mathrm{C}$, à estimer le potentiel de rendement de nos meilleurs haricots à $90 \%$ de celui des meilleurs Vigna sélectionnés sur place. 


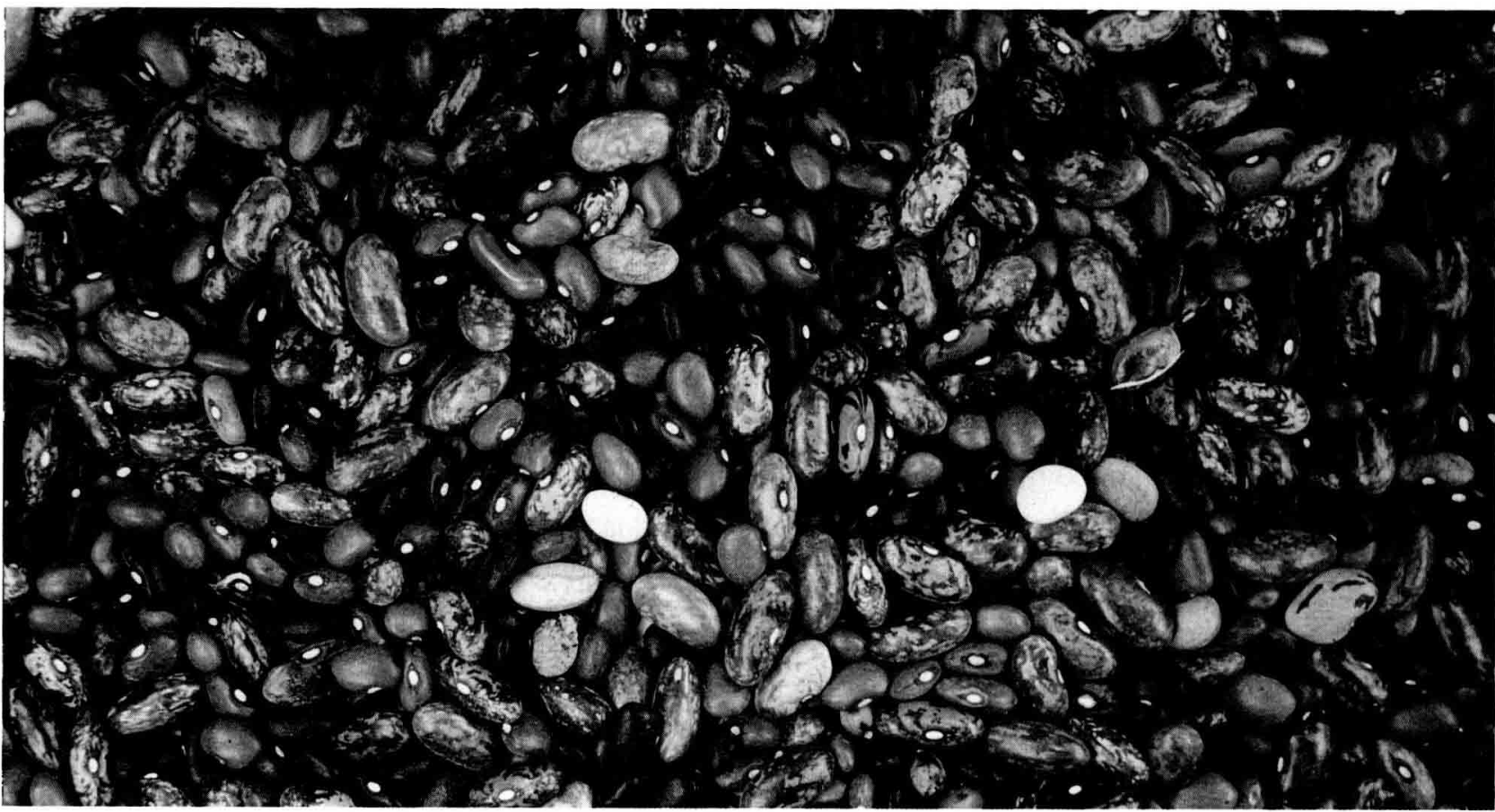

Fig 2. Mélange variétal cultivé par un paysan du Plateau Salagnac.

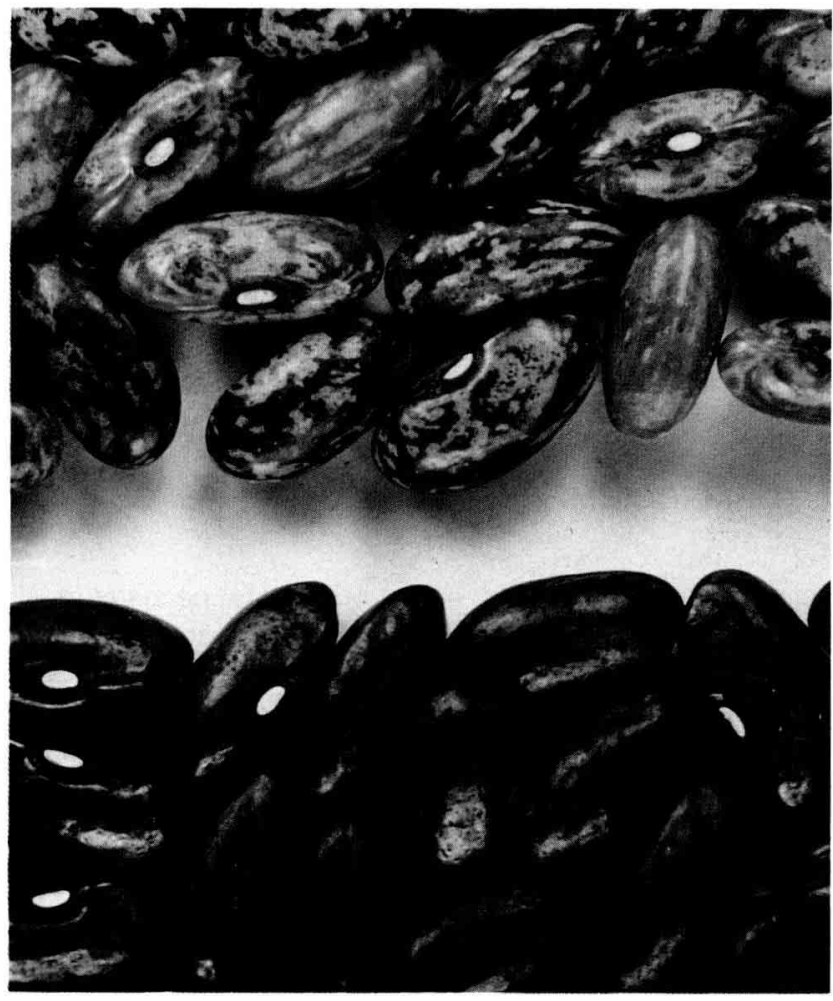

Fig 3. Coloration comparee de "Salagnac 86" (en haut) et de "Salagnac 90" (en bas).

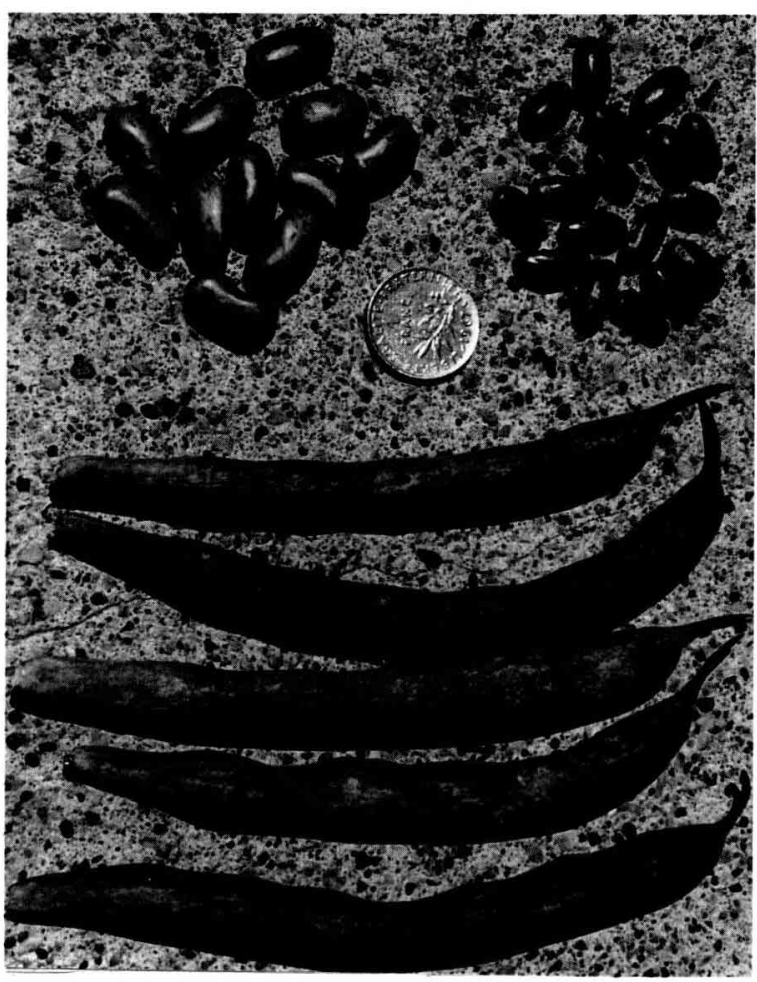

Fig 4. La lignee "CNR 11» gousse grouge vif uni, grains écosses frais à gaucne, grains secs à droite. La pièce française de un franc donne l'écnelle. 


\section{INTÉRÊT DES LIGNÉES ISSUES DE GÉNITEURS HAITIENS HORS DE LA ZONE ANTILLAISE}

Nous avons pu disposer de correspondants pour réaliser des essais variétaux :

- à la Grande Comore où en 1985-1986 les lignées B801, Kenscoff super, Nicanor 2.4 (non tuteurées) et Cap Haïtien 9.4 (tuteurée) ont donné de bons résultats à $600-800 \mathrm{~m}$ d'altitude;

- à la Réunion, dans des essais réalisés par Michelon (IRAT) dans le cadre "diversification des productions dans la zone de culture du géranium odorant" (altitude de 800-1 $000 \mathrm{~m}$ ). On peut citer les résultats présentés tableau $\mathrm{I}$.

Les récoltes de Salagnac 90, équivalentes à celles des meilleures variétés rwandaises, ont été obtenus avec 20-25 j d'avance sur ces dernières.

Tableau I. Production de différentes variétés cultivées à la Réunion.

\begin{tabular}{|c|c|c|c|}
\hline Variétés & Origine & $\begin{array}{c}\text { Moyenne } \\
\text { de } 3 \text { essais } \\
\text { en station } \\
\text { (tha) }\end{array}$ & $\begin{array}{c}\text { Moyenne } \\
\text { d'essais en } \\
\text { conditions } \\
\text { paysannes } \\
\text { (tha) }\end{array}$ \\
\hline Salagnac 90 & Haïti & 0,90 & 0,91 \\
\hline Kenscoff super & & 0,77 & - \\
\hline Emma & & 0,88 & - \\
\hline Rubona & ISAR-Rwanda & 0,88 & 0,96 \\
\hline $\operatorname{var} n^{\circ} 11$ & & 0,84 & 0,87 \\
\hline Pompadour & $\begin{array}{l}\text { République } \\
\text { dominicaine }\end{array}$ & 0,79 & 0,88 \\
\hline Marlat & Réunion & 0,64 & 0,42 \\
\hline
\end{tabular}

Tableau II. Récolte (tha) obtenue pour différentes variétés cultivées au Burkina-Faso.

Semis Semis

le 10 janvier 1986 le 23 février 1987

$\begin{array}{lcc}\text { Kenscoff super } & 1,60 & 1,57 \\ \text { Salagnac } 90 & 1,29 & 0,96 \\ \text { CNR } 11 & 1,33 & -\end{array}$

En conditions de plaine, au Burkina-Faso, d'Arondel de Hayes (IRAT) a, au contraire, obtenu de meilleurs résultats avec Kenscoff super (récoltes en t/ha) (tableau II).

Signalons enfin que dans le midi de la France, nous avons retrouvé pour Salagnac 90, (C1 $x$ I)4.3, Kenscoff super, Azaël x S862, CNR.11, CNR.44, Salagnac 59, CGR.I et CGR.D une productivité analogue à celle que nous observions en Guadeloupe. Une production de semences de ces variétés est donc possible en conditions Nord-méditerranéennes, avec un cycle allongé de 15-20 j par rapport à la Guadeloupe, du fait des températures basses en début de cycle pour les semis de fin avril ou début mai. Cela nous a conduit à proposer CNR.11 et CNR.44 pour l'inscription au catalogue des variétés qui ne comporte, comme haricot rouge, que Rognon de Coq, variété aujourd'hui désuète.

\section{CONCLUSION}

Sans réaliser le grand «bond en avant» vers des rendements de 3 ou 3,5 t/ha en haricots secs, qui constitue un des objectifs principaux du CIAT, nous avons obtenu, dans la descendance de croisements à partir des meilleures lignées haïtiennes, des variétés à cycle court pouvant donner satisfaction dans des climats très variés.

Ce résultat est dû sans doute principalement aux qualités de précocité et d'indifférence à la longueur du jour du matériel de départ, mais peut-être aussi également à la politique de sélection en générations alternées dans des lieux différents, appliquée d'abord au choix des géniteurs, ensuite au processus de sélection dans les descendances de croisement.

\section{REMERCIEMENTS}

Un générique de 14 noms aurait été nécessaire si nous avions voulu citer, comme auteurs, tous ceux qui ont contribué aux résultats mentionnés dans cette publication, nous les mentionnerons ici :

- $\mathrm{H}$ Bannerot et G Fouilloux (INRA, Versailles, génétique et amélioration des plantes), nos «tuteurs scientifiques";

- G Jacqua, P Pauvert, F Bannis (INRA, station de pathologie végétale, Antilles-Guyane);

- C Vincent et son équipe (INRA, station d'amélioration des plantes Antilles-Guyane);

- $M$ Brochet, $V$ de Reynal, $P$ Lucas (coopérants français); 
- P Mathieu, A Laraque, L Pierre-Jean (agronomes haïtiens) du projet Madian-Salagnac;

- MM Michelon et d'Arondel de Hayes (IRAT, Réunion et Burkina-Faso) ainsi que tous les Haïtiens et Haïtiennes rencontrés dans leurs champs ou sur les marchés;

- l'ambassadeur de France et la mission de Coopération française en Haïti, et les professeurs de la faculté d'agronomie et de médecine vétérinaire de Damien.

Nos recherches ont été subventionnées par le contrat TSDA 073F de la commission des Communautés européennes de 1985 à 1988 .

\section{RÉFÉRENCES}

Belliard-Alonzo LM (1971) Contribución al estudio de les enfermedades de la habichuela ( $P$ vulgaris) en la Republica dominicana. Tésis Universidad autonoma de Santo Domingo
Fouilloux G (1978) Méthodes permettant l'accélération des processus de sélection chez les plantes potagères. Sél Fr 25, 9-19

Messiaen CM, Pauvert $P$, Jacqua $G$, Laraque A (1989) L'oïdium américain du haricot (Erysiphe polygoni) dans la zone antillaise : recherche de géniteurs de résistance. Agronomie 9, 259-263

Pauvert $P$ (1989) Contribution à l'étude des races d'oïdium du haricot (E polygoni) en Guadeloupe. Agronomie 9, 265-269

Prakken R (1972) Seed coat color in Phaseolus vulgaris, attempt to a general synthesis. Ann Rpt Bean Improv Coop 74-78

Schwartz HF, Galvez GE (1978) Problemas de campo de los cultivos de frijol en America latina. CIAT, $136 \mathrm{p}$

Toro O, Tohme J, Debouck DG (1990) Wild bean (Phaseolus vulgaris L) description and distribution. International Board for Genetic Resources (IPBGR) and Centro Internacional de Agricultura Tropical (CIAT) Cali, Colombia $106 \mathrm{p}$ 